\title{
Multi-modular scalable DC-AC power converter for current injection to the grid based on predictive voltage control
}

\author{
S. Toledo and M. Rivera \\ Dept. of Electrical Engineering \\ Univerisdad de Talca \\ Curicó, Chile \\ \{stoledo,mrivera $\} @$ utalca.cl
}

\author{
E. Maqueda, M. Ayala, J. Pacher, C. Romero \\ and R. Gregor \\ Engineering Faculty \\ Universidad Nacional de Asunción \\ Luque, Paraguay \\ \{emaqueda,mayala,jpacher,cromero,rgregor\}@ing.una.py \\ P. Wheeler \\ Dept. of Electrical and Electronic Engineering \\ University of Nottingham \\ Nottingham, UK \\ pat.wheeler@nottingham.ac.uk
}

\author{
T. Dragicevic \\ Dept. of Energy Technology \\ Aalborg University \\ Aalborg, Denmark \\ tdr@et.aau.dk
}

\begin{abstract}
Increasing world electrical energy demand make necessary to develop new efficient and reliable schemes for power injection to the existing grid under distributed generation frames. In this work a novel DC-AC control scheme is proposed, which is based on one hand, on several 2L-VSI working together as a multi-modular converter in a parallel switching connection topology in the power converter stage and, in the other hand, a control strategy using two loops, an internal based on predictive voltage control and another external applying a PR current control, achieving THD levels lower than $1 \%$ for the injected current, accomplishing with the international standards for these kind of applications.
\end{abstract}

Index Terms-Current injection, distributed generation systems, multi-modular VSI, parallel switches, predictive voltage control.

\section{INTRODUCTION}

$\mathbf{I}$ $\mathrm{N}$ the last decades, different renewable energies, such as: micro-hydro, solar, wind farms, etc [1]-[3], have been studied in order to use them in isolated or grid-on electric systems. In every case, power converters are often used as a fixed power source [4]-[6]. These converters have to secured a controlled active and reactive power flux, reduce the harmonic content in currents and voltages, reduce the resonance effect and secure a good grid synchronization, in case of distributed systems. To accomplish these requirements, different control techniques have been studied, such as proportional integral (PI) control [7], proportional resonant (PR) control [8], predictive control [9], etc, and in many cases, they are combined

The authors express their gratitude to Consejo Nacional de Ciencia y Tecnología de Paraguay (CONACYT), for the support and financing through Project PINV15-0584, to CONICYT of Chile through the FONDECYT Regular Project 1160690, Project MEC 80150056 and CONICYTPFCHA/Doctorado Nacional/2019-21192003. with modulation techniques, such as: pulse width modulation (PWM) [10], space vector modulation (SVM) [11]-[13], etc. However, the majority of power converters are commonly used as a fixed power source, which complicates the scalability in terms of energy conversion system, mainly in applications where an extension of the system, based on renewable energies, is planned in order to respond efficiently to an increase in demand associated with a variation in load requirements.

This paper presents the design of the architecture of a multimodular power converter to obtain scalable power, where each module consists in a three-phase voltage source inverter (VSI), with a novel control scheme based on two close loops, which consist in an internal predictive voltage control and an outer PR current control. This feature is interesting in applications where power converters with reduced volume, high energy density and a good availability are required.

This paper is organized as follows: the proposed current control scheme is shown in Section II. In Section III, the model predictive control (MPC) applied as a voltage control is presented. Section IV describes the reference voltage generator stage. Section V depicted some experimental results for parallel connected switches. The grid connection process are described in Section VI. Obtained simulation results are shown in Section VII regarding transient and steady-state performance. Finally, conclusions are presented in the last section.

\section{Proposed CURRENT CONTROL SCHEME}

As it is shown in Fig. 1, the proposed control scheme consists of several two level VSI (2L-VSI) modules working together, where every switch is connected in parallel with the corresponding switches on other VSI dividing the circulating 
currents by " $n$ " (where $n$ represents the number of modules) allowing, for example, the increase of power capability of the whole system, or to decrease the charge for every leg allowing the use of smaller semiconductors for the same rated power. In this case, the switching signal is the same for every module, that is, the same commutation pattern is applied in every VSI connected in parallel such as the commutation occurs at the same time achieving a multi-tracking configuration for the circulating current. The mentioned configuration will be treated in a more detailed way in Section V and some experimental results using parallel switches will be depicted in terms of the circulating currents behavior in order to validate the proposed scheme.

The DC source comes from a $2.4 \mathrm{~kW}$ solar photo-voltaic (PV) system at the input of the power converter and a LC filter at the output being the capacitor voltage $\left(v_{o}\right)$ the output signal that must be controlled in order to achieve an interconnection to the grid. A bypass switch is needed to connect the generation system with the grid in the common coupling point (PCC) once both stages were synchronized. The control consists of an inner predictive voltage control (PVC) stage with a fast transient response and an outer PR current control plus a feed-forward control to generate the reference for the voltage control stage. The feed-forward control comes from a voltage reference generator that takes the phase of the grid voltage $\left(v_{g}\right)$ from a phase-locked loop (PLL) generating a synchronized signal with the grid as the desired output of the converter stage. At the beginning, the desired injected grid currents $\left(i_{g}\right)$ must to be set to zero as well as $v_{g}$ and $v_{o}$ should be the same, then the PR output will be zero at the starting point. Once both voltages have been synchronized, the bypass connects the generation stage with the grid and the current can be controlled by setting the desired current at the input of the PR controller. The PVC selects the optimal switching combination, in every sampling time, to control the output capacitor voltage that generates the desired current injection. In the next section the implemented PVC technique is depicted.

\section{Predictive Voltage Control}

MPC strategy consists on predicting the future behaviour of the analyzed system for each possible input state based on dynamic equations which define it, and picking the best state which fulfills the desired output. In this way, the first step to apply this control is to obtain the system model.

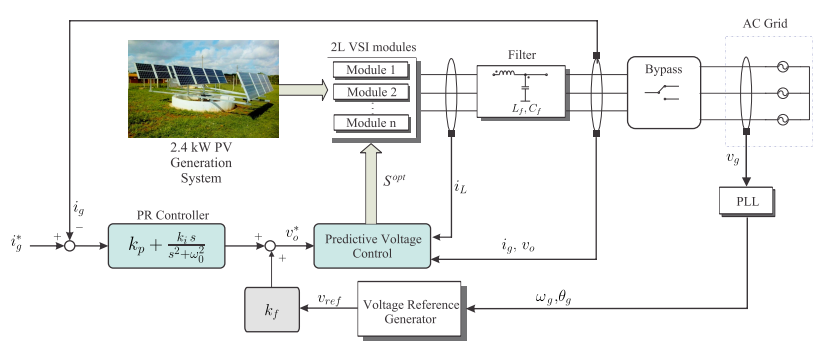

Fig. 1. Proposed control scheme.

\section{A. Power Converter Model}

The scheme of a basic module of the converter is shown in Fig. 2, which is composed of a DC source, a 2L-VSI and an output $L C$ filter.

The $2 \mathrm{~L}-\mathrm{VSI}$ is one of the most used topologies in the literature [13]-[15]. In this case, it is possible to define a switching function as $S_{x} \in\{1,0\}$ where $x$ denotes each phase $(x=a, b, c)$. Given that each leg is conformed by two switches that should be activated in a complementary form to avoid short-circuits between the terminals of the DC source $v_{d c}$, the valid switching states are the following:

$$
\begin{gathered}
S_{a}= \begin{cases}1, & \text { if } S_{1}=1 \text { and } S_{4}=0, \\
0, & \text { if } S_{1}=0 \text { and } S_{4}=1,\end{cases} \\
S_{b}= \begin{cases}1, & \text { if } S_{2}=1 \text { and } S_{5}=0, \\
0, & \text { if } S_{2}=0 \text { and } S_{5}=1,\end{cases} \\
S_{c}= \begin{cases}1, & \text { if } S_{3}=1 \text { and } S_{6}=0, \\
0, & \text { if } S_{3}=0 \text { and } S_{6}=1 .\end{cases}
\end{gathered}
$$

Only eight switching states are valid for the 2L-VSI. Likewise, it is possible to synthetize the voltages at the points $a, b$ and $c$ regarding the point $N\left(v_{a N}, v_{b N}\right.$ y $\left.v_{c N}\right)$ depending on the switches of the 2L-VSI and $v_{d c}$ as:

$$
v_{a N}=S_{a} v_{d c}, \quad v_{b N}=S_{b}, v_{d c}, \quad v_{c N}=S_{c} v_{d c} .
$$

In order to determine the effective voltages applied for each phase (i.e., from $a, b, \mathrm{y} c$ to $n$ ), the common mode voltage $v_{n N}$ must be subtracted from the equation (2). It is possible to determine $v_{n N}$ in a simple way by applying Kirchhoff voltage law as follows:

$$
v_{n N}=\frac{v_{a N}+v_{b N}+v_{c N}}{3} .
$$

So, the effective phase voltage is given by:

$v_{a n}=v_{a N}-v_{n N}, \quad v_{b n}=v_{b N}-v_{n N}$ and $v_{c n}=v_{c N}-v_{n N}$.

By applying Clarke's transformation [16] to each valid state of the 2L-VSI module, it is possible to represent the space vector graph which is depicted on Fig. 3 showing the eight valid voltage vectors in the $\alpha-\beta$ plane.

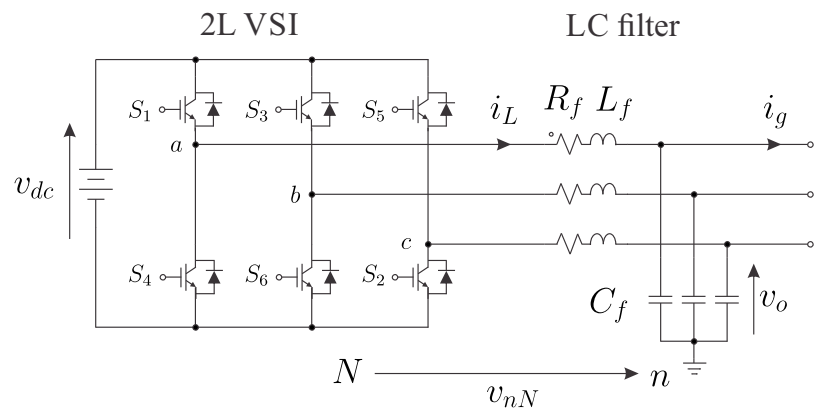

Fig. 2. Conversion module topology. 


\section{B. Output Filter Model}

As can be seen in Fig. 2, the 2L-VSI module is connected to a load through an $L C$ output filter. Each converter leg possess an inductor $L_{f}$ and its corresponding leakage resistance $R_{f}$ and a capacitor $C_{f}$. By considering the inductor currents $i_{L}$ and the capacitor voltage $v_{o}$ as state variables of this second order system and assuming that the parameters are equal for the three legs, the system dynamic in $\alpha-\beta$ plane is given by:

$$
L_{f} \frac{d i_{L \alpha \beta}}{d t}=v_{\alpha \beta}-v_{o \alpha \beta}-R_{f} i_{L \alpha \beta},
$$

where $v_{\alpha \beta}$ corresponds to one of the valid vectors shown in Fig. 3. On the other hand, the dynamic behaviour of the capacitor voltage is defined as follows:

$$
C_{f} \frac{d v_{o \alpha \beta}}{d t}=i_{L \alpha \beta}-i_{g \alpha \beta} .
$$

In this way, the state space representation of the system is:

$$
\frac{d \mathbf{x}}{d t}=\mathbf{A} \mathbf{x}+\mathbf{B u}
$$

where

$$
\begin{gathered}
\mathbf{x}=\left[\begin{array}{c}
i_{L \alpha \beta} \\
v_{o \alpha \beta}
\end{array}\right], \quad \mathbf{A}=\left[\begin{array}{cc}
-\frac{R_{f}}{L_{f}} & -\frac{1}{L_{f}} \\
\frac{1}{C_{f}} & 0
\end{array}\right], \\
\mathbf{u}=\left[\begin{array}{c}
v_{\alpha \beta} \\
i_{g \alpha \beta}
\end{array}\right] \mathrm{y} \quad \mathbf{B}=\left[\begin{array}{cc}
\frac{1}{L_{f}} & 0 \\
0 & -\frac{1}{C_{f}}
\end{array}\right] .
\end{gathered}
$$

The previous equations define completely the continuous model for the $L C$ filter by considering the output voltages of the 2L-VSI $v_{\alpha \beta}$ and the load currents $i_{g \alpha \beta}$ as inputs.

\section{Discrete Time Model of LC Filter}

The discrete model of the system is given by:

$$
\mathbf{x}(k+1)=\mathbf{A}_{d} \mathbf{x}(k)+\mathbf{B}_{d} \mathbf{u}(k),
$$

being $\mathbf{A}_{d}=e^{\mathbf{A} T_{s}}, \mathbf{B}_{d}=\int_{0}^{T_{s}} e^{\mathbf{A}\left(T_{s}-\tau\right)} \mathbf{B} d \tau$ and $T_{s}$ corresponding to the sampling time. Thus, by using a discrete model that describes the system it is possible to predict the states of $v_{o}$ e $i_{L}$ as follows:

$$
i_{L}(k+1)=a_{11} i_{L}(k)+a_{12} v_{o}(k)+b_{11} v(k)+b_{12} i_{g}(k),
$$

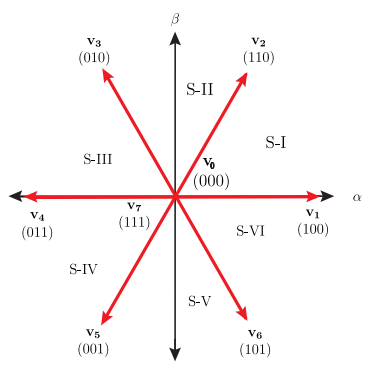

Fig. 3. Valid vectors $\alpha-\beta$ plane.

$$
v_{o}(k+1)=a_{21} i_{L}(k)+a_{22} v_{o}(k)+b_{21} v(k)+b_{22} i_{g}(k),
$$

siendo

$$
\mathbf{A}_{d}=\left[\begin{array}{ll}
a_{11} & a_{12} \\
a_{21} & a_{22}
\end{array}\right], \mathbf{B}_{d}=\left[\begin{array}{ll}
b_{11} & b_{12} \\
b_{21} & b_{22}
\end{array}\right],
$$

where $k$ is the actual instant time and $k+1$ indicates the beginning of the next sample instant. Through the previous equations it is possible to predict the inductor currents values and the voltage output to implement the MPC.

\section{Predictive Voltage Control}

The basic operating principle of the algorithm is the following: first, at the beginning of the sampling instant, new measurements of $v_{o}, i_{L}$ and $i_{g}$ are obtained. These measurements define the starter point from which the algorithm predicts the future trajectory of the state space variables by considering (10) and (11), for each feasible voltage vector. Every predicted value is evaluated with a pre-designed cost function (CF), and the vector with the lowest $\mathrm{CF}$ is applied to the $2 \mathrm{~L}-\mathrm{VSI}$ switches. The presented technique, in this section, is based on the proposed control in [17], [18]. The selected cost function is the following:

$$
g=\left(v_{o \alpha}^{*}-v_{o \alpha}\right)^{2}+\left(v_{o \beta}^{*}-v_{o \beta}\right)^{2}+\lambda_{d} g_{d e r},
$$

being $v_{o \alpha}^{*}$ and $v_{o \beta}^{*}$ the desired voltages on the $\alpha-\beta$ plane and defining:

$$
g_{d e r}=\left(C_{f} \omega_{r e f} v_{o \beta}^{*}-i_{L \alpha}+i_{g \alpha}\right)^{2}+\left(C_{f} \omega_{r e f} v_{o \alpha}^{*}-i_{L \beta}+i_{g \beta}\right)^{2} .
$$

The term $g_{d e r}$ is introduced with the purpose of correcting the incapacity of the classic predictive control of tracking the capacitor voltage derivative resulting in a high total harmonic distortion (THD), creating an ideal regulator which controls the voltage capacitor and its derivative effectively. The effect of the derivative term is controlled with a weighting factor $\lambda_{d}$.

This strategy is based on the evaluation, at every sampling instant, of the cost function $g$ for all the valid vectors and to apply the vector which minimizes the CF in the next sampling instant achieving a desired voltage tracking.

\section{OUTER CURRENT CONTROL LOOP FOR REFERENCE VOLTAGE GENERATION}

As mentioned above, the proposed scheme is based on the implementation of two control loops, an internal loop corresponding to the predictive voltage control and an external loop that generates the voltage reference to inject a controlled current into the grid. In Fig. 1 the external control loop is shown where the difference between the injected current $i_{g}$ and the desired current $i_{g}^{*}$ is calculated to be applied as the input of a PR controller [19] which output is added to a feedforward control signal $v_{\text {ref }}$ which is used at system startup to synchronize the output voltage of the conversion stage $v_{o}$ with the grid voltage $v_{g}$ so as to be connected through a bypass. This generated reference voltage is the input to 
the voltage predictive control block to calculate the optimum vector to be applied. The voltage $v_{r e f}$ is generated from the measurement of the phase of the grid $\theta$ obtained from the PLL and the amplitude of the signal of the grid. The value of $i_{g}^{*}$ is determined from the current amplitude and phase according to the desired active and reactive power.

Once the desired currents generate the desired voltages and the predictive voltage controller selects the vector to be applied, the same commutation signals are applied to every module generating several paths for the current to be splitted from the total flow decreasing the thermal stress and providing an increased power management capability. In order to validate the idea of using the same commutation signal to control parallel switches to split the circulating current, in next section experimental tests of the proposed parallel topology are depicted.

\section{EXPERIMENTAL TEST OF THE PROPOSED PARALLEL SWITCHES TOPOLOGY}

The proposed topology implies the application of the same switching signal to every module of the converters hoping that the current splits to every path and the reaction to contingencies is based on the capability of the system to manage the additional current in case of broken switch for example. In this section a brief study of the behavior of parallel switches is presented.

In order to verify the parallel operation of several modules of the VSI converter, an experimental test bench has been designed. The test circuit can be seen in Fig. 4. The circuit shows the modular interconnection of the first branch of three identical VSI converters connected in parallel. Each branch is composed of two switches in parallel and in the same way each switch of the converter branch is parallel to each other. The circuit is connected at the input to a DC voltage source and at the output to a resistive load. By means of this design, an analysis is carried out that consists of controlling the switches through the same signal for each VSI converter where the behavior of the current delivered by the first branch of each converter is studied to show how the flow is splitted and how it behaves under faults in any switch.

Fig. 5 shows the setup test implemented in order to evaluate the operation of several modules of the VSI converters in parallel connection. The figure shows the electronic boards of each branch of the converters, in modular form, the DC input source and an oscilloscope. The main components, used in the implementation of the VSI converter, are: a) for the switch the $\mathrm{SiC}$ MOSFET power semiconductor CAS120M12 from the

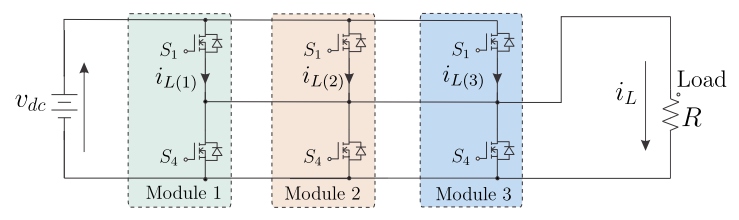

Fig. 4. Tests circuit diagram.

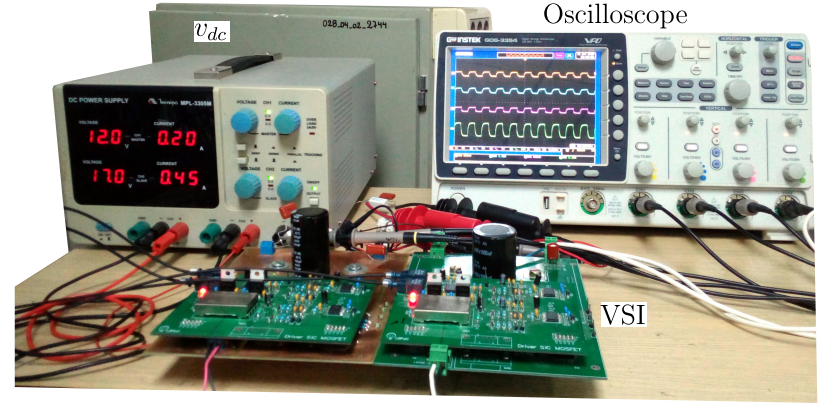

Fig. 5. Experimental test setup.

manufacturer CREE, b) the IR2110 driver for the control of the trip signal of the switches, and c) fiber optic transceivers HFBR-0500Z for signal reception of programmable control devices. In the circuit the measurements of the output current of each branch of the converters were made, represented in Fig. 4 by $i_{L(1)}, i_{L(2)}$ e $i_{L(3)}$ respectively. The sum of these currents is represented by $i_{L}$ and it corresponds in turn to the current that passes through the resistance $R$.

The waveforms of the currents $i_{L(1)}, i_{L(2)}, i_{L(3)}$ e $i_{L}$ can be seen in Fig. 6. It is observed that initially each current of each branch of the converters are very similar, $2.5 \mathrm{~A}$ pkpk respectively, while the total current $i_{L}$ displayed by the channel 4 of the oscilloscope showing a value of $7.5 \mathrm{~A} \mathrm{pk-pk}$ (i.e. three times the current in every branch as is expected), being an expected behavior. Further on, it is possible to observe the moment of fault over the path of the current $i_{L(3)}$, corresponding to the extraction of the third VSI converter of the system. At that instant, the magnitude of the current $i_{L(3)}$ is evenly distributed in the currents $i_{L(1)}$ and $i_{L(2)}$ as shown in Fig. 6, and also, for the transient, the current $i_{L}$ over the load $R$ does not present disturbances.

These results allow to confirm the feasibility of using parallel switching configuration with the same commutation signal achieving a multi-tracking operation that can manage

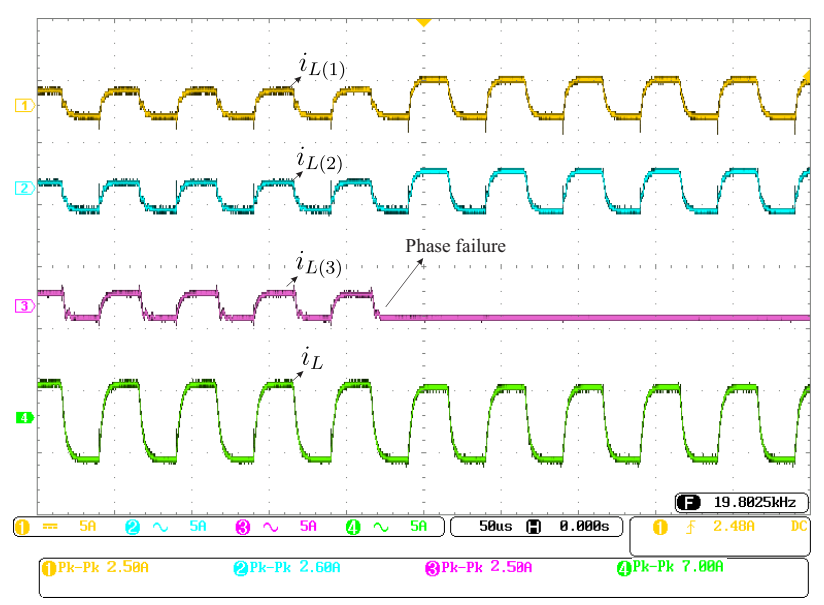

Fig. 6. Switching waveform of the current of three branches of the VSI in parallel. 


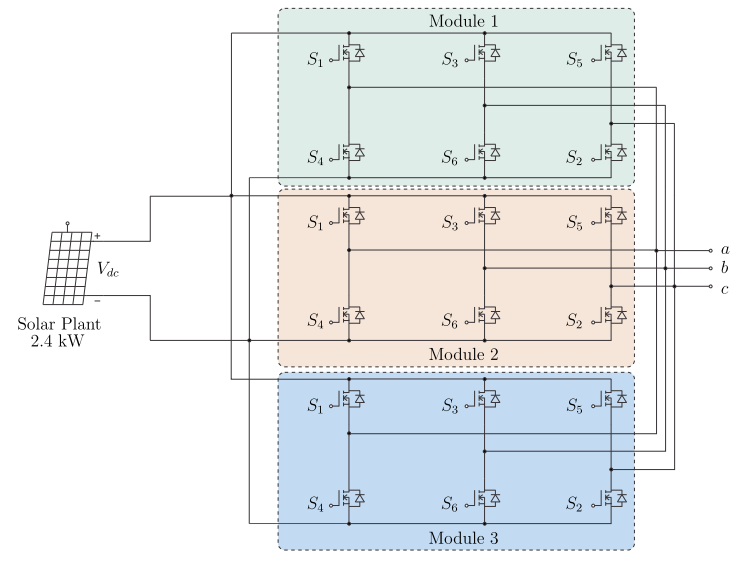

Fig. 7. multi-modular DC-AC converter scheme.

contingencies and even increase the power supplied capability of the whole system adding more modules depending on the demanded energy.

Fig. 7 shows the multi-modular configuration for three parallel VSIs that will be used on the final implementation of the proposed grid connected generation system.

\section{GRID CONNECTION PROCESS}

The grid connection process is performed as follows:

1) The predictive voltage control is used to generate an output voltage $\left(v_{o}\right)$ synchronized with the grid voltage $\left(v_{g}\right)$, that is, the voltage of the capacitor is synchronized with the grid in order to be connected on the CCP.

2) Next, once the voltages are equal, the bypass is used to connect the systems with the reference current $\left(i_{g}^{*}\right)$ set to zero.

3) Finally, the feed-forward signal can be set to zero and all the reference voltage comes from the PR controller, then it is possible to select the desired injected current $\left(i_{g}\right)$.

Based on the above described procedure, it is possible to evaluate the proposal performing some simulations using MATLAB/Simulink environment.

\section{SimUlation RESUlTS OF THE PROPOSED GRID CONNECTED GENERATION SYSTEM}

The multi-modular grid connected generation system consists on three modules (i.e. as shown in Fig. 7) under the control scheme depicted on Fig.1 and with the parameters of Table I. In Fig. 8 the interconnection process and the behavior under steps of desired currents are shown. At the beginning, the injected current is zero because the generation system is not connected to the grid and the output voltage $\left(v_{o}\right)$ is still in synchronization process regarding the grid voltage $\left(v_{g}\right)$. This process takes around 0.14 seconds. After 0.2 seconds, the bypass connects the output capacitors with the grid but $i_{g}$ is still set to zero generating a short transient during the connection instant. Then the system is ready to inject the
TABLE I

SIMULATION PARAMETERS.

\begin{tabular}{lccc}
\hline & \multicolumn{3}{c}{ Simulation parameters } \\
\cline { 2 - 4 } Parameter & Symbol & Value & Unit \\
\hline \hline Grid voltage & $v_{g}$ & 311 & $V_{p}$ \\
Grid frequency & $f_{g}$ & 50 & $\mathrm{~Hz}$ \\
DC link voltage & $v_{d c}$ & 700 & $\mathrm{~V}$ \\
Leakage inductor resistance & $R_{f}$ & 10 & $\mathrm{~m} \Omega$ \\
Filter inductance & $L_{f}$ & 2.4 & $\mathrm{mH}$ \\
Filter capacitance & $C_{f}$ & 24 & $\mu \mathrm{F}$ \\
Sampling time & $T_{s}$ & 25 & $\mu s$ \\
Sampling frequency & $f_{s}$ & 40 & $\mathrm{kHz}$ \\
Derivative control weighting & $\lambda_{d}$ & 0.2 & \\
PR proportional control constant & $k_{p}$ & 10 & \\
PR integral control constant & $k_{i}$ & 1500 & \\
\hline
\end{tabular}
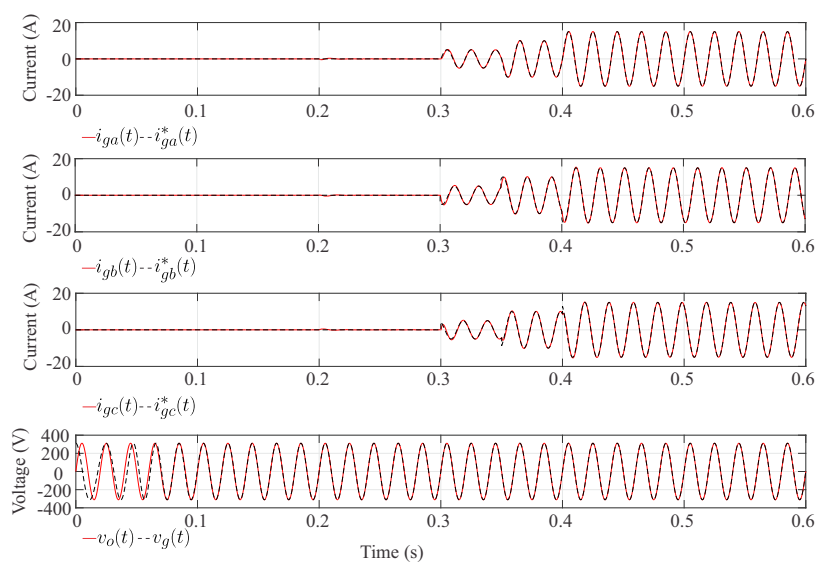

Fig. 8. Injected current and output voltage of the proposed control scheme.

desired current (at this point it is possible to set the feedforward control to zero gradually changing $k_{f}$ from 1 to 0 , being careful not to generate high current pikes). At 0.3 seconds the desired current $i_{g}^{*}$ is set to $5 \mathrm{amps}$, then at 0.35 seconds is set to 10 amps and finally, at 0.4 seconds is set to 15 amps. During this current steps it is possible to appreciate a good tracking of the reference. The THD oscillates from 0.33 to $0.9 \%$ in the injected current. The tracking error during the input step, applied at 0.4 seconds, is shown in Fig. 9 where the transient stage can be measured to be around 30 milliseconds approximately. That is due to the use of the PR controller in the outer loop. In this case it is possible to use the inner predictive voltage control since this technique presents a fast transient response compared with the PR controller.

Besides the current tracking test, an under failure test has been performed and the results are reflected in Fig. 10. In this Figure it is possible to appreciate the behavior of the multi-modular converter at the moment of a failure occurs. As it can be seen, the circulation current is homogeneously distributed between every switch (some minimal differences can be found in the experimental implementation due to the parametric variation between different Sic-MOSFET devices). $i_{L 1}, i_{L 2}$ and $i_{L 3}$ correspond to the circulating current through modules 1,2 and 3 , respectively. $i_{L}$ corresponds to the total current supplied by the converter to the output filter and finally, 


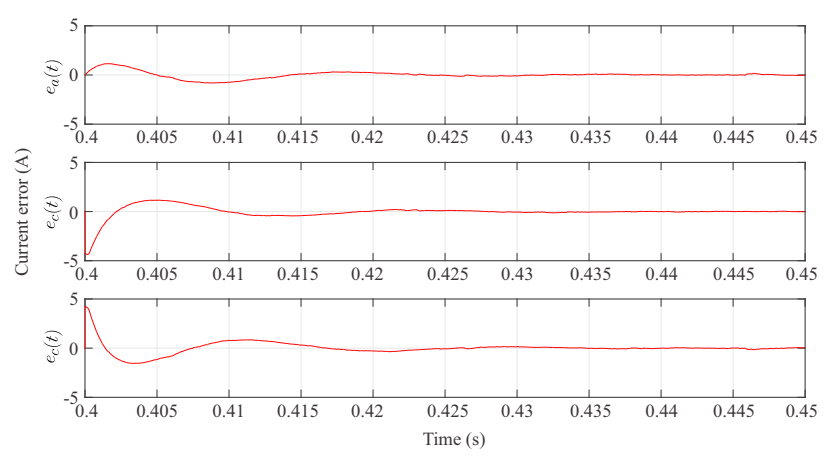

Fig. 9. Current error under input steps.

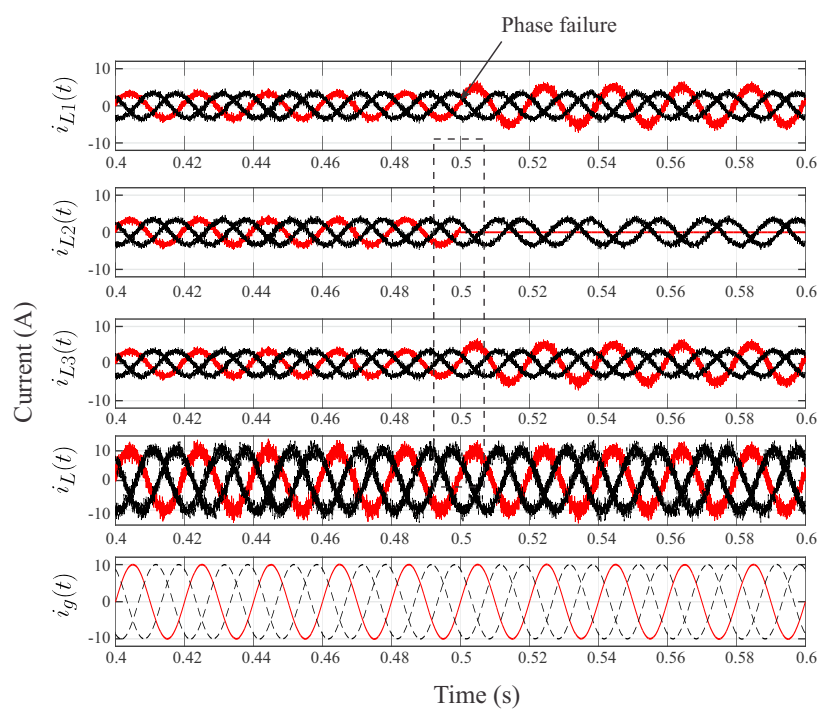

Fig. 10. Current behavior under a phase failure.

$i_{g}$ is the total injected current. At 0.5 seconds, a failure in the module 2 is simulated, it is seen that the path was broken and $i_{L 2}$ goes to zero. In order to hold the injected current $i_{g}$, the current splits between $i_{L 1}$ and $i_{L 3}$ such a way that the effect of this disconnection is negligible. These results are consistent with those observed in the experimental tests of the section V. Concerning the voltage, it presents a THD around $0.77 \%$ that is a good value, this due to the derivative control term added to the classic PVC.

\section{CONCLUSION}

The main contribution of this paper lies on the proposal of a novel injected current control strategy based on two control loops with a multi-modular VSI converter topology. The multi-modular topology provides more energy availability due to the possibility of splitting the current between several paths at the moment that a failure occurs. Moreover, this topology provides in turn scalability in the implementation due to the parallel topology shows a good performance sharing the same commutation signal which means it is feasible to connect the necessary number of modules in order to provide and manage the desired power. The control strategy shows a good performance for current tracking, error signals and THD reduction, accomplishing with all the international standards related to this kind of application.

\section{REFERENCES}

[1] W. Xiao, M. S. El Moursi, O. Khan, and D. Infield, "Review of gridtied converter topologies used in photovoltaic systems," IET Renewable Power Generation, vol. 10, no. 10, pp. 1543-1551, 2016.

[2] Ö. Göksu, R. Teodorescu, C. L. Bak, F. Iov, and P. C. Kjær, "Instability of wind turbine converters during current injection to low voltage grid faults and pll frequency based stability solution," IEEE Trans. Power Systems, vol. 29, no. 4, pp. 1683-1691, 2014.

[3] S. Toledo, M. Rivera, and J. L. Elizondo, "Overview of wind energy conversion systems development, technologies and power electronics research trends," in 2016 IEEE International Conference on Automatica (ICA-ACCA), Oct 2016, pp. 1-6.

[4] A. Lesnicar and R. Marquardt, "An innovative modular multilevel converter topology suitable for a wide power range," in 2003 IEEE Bologna Power Tech Conference Proceedings,, vol. 3. IEEE, 2003, pp. $1-6$.

[5] M. Hagiwara and H. Akagi, "Control and experiment of pulsewidthmodulated modular multilevel converters," IEEE Trans. Power Electron., vol. 24, no. 7, pp. 1737-1746, 2009.

[6] A. Antonopoulos, L. Angquist, and H.-P. Nee, "On dynamics and voltage control of the modular multilevel converter," in 2009 13th European Conference on Power Electronics and Applications. IEEE, 2009, pp. $1-10$.

[7] V. Valouch, M. Bejvl, P. Šimek, and J. Škramlík, "Power control of grid-connected converters under unbalanced voltage conditions," IEEE Trans. Ind. Electron., vol. 62, no. 7, pp. 4241-4248, 2014.

[8] D. Pérez-Estévez, J. Doval-Gandoy, A. G. Yepes, O. Lopez, and F. Baneira, "Enhanced resonant current controller for grid-connected converters with lcl filter," IEEE Trans. Power Electron., vol. 33, no. 5, pp. 3765-3778, 2017

[9] D. Caballero, S. Toledo, M. Rivera, E. Maqueda, F. Gavilan, C. Romero, and R. Gregor, "Predictive voltage control using matrix converter for a stand-alone wind energy based microgrid," in 2018 IEEE International Conference on Automation/XXIII Congress of the Chilean Association of Automatic Control (ICA-ACCA). IEEE, 2018, pp. 1-6.

[10] J. A. Riveros, J. Prieto, M. Rivera, S. Toledo, and R. Gregor, "A generalised multifrequency pwm strategy for dual three-phase voltage source converters," Energies, vol. 12, no. 7, p. 1398, 2019.

[11] M. Rivera, S. Toledo, C. Baier, L. Tarisciotti, P. Wheeler, and S. Verne, "Indirect predictive control techniques for a matrix converter operating at fixed switching frequency," in 2017 IEEE International Symposium on Predictive Control of Electrical Drives and Power Electronics (PRECEDE). IEEE, 2017, pp. 13-18.

[12] O. Gonzalez, M. Ayala, J. Doval-Gandoy, J. Rodas, R. Gregor, and M. Rivera, "Predictive-fixed switching current control strategy applied to six-phase induction machine," Energies, vol. 12, no. 12, p. 2294, 2019.

[13] F. Gavilan, D. Caballero, S. Toledo, E. Maqueda, R. Gregor, J. Rodas, M. Rivera, and I. Araujo-Vargas, "Predictive power control strategy for a grid-connected 21-vsi with fixed switching frequency," in Power, Electronics and Computing (ROPEC), 2016 IEEE International Autumn Meeting on. IEEE, Nov 2016, pp. 1-6.

[14] S. Wang, C. Li, C. Che, and D. Xu, "Direct torque control for 21-vsi pmsm using switching instant table," IEEE Trans. Ind. Electron., vol. 65, no. 12, pp. 9410-9420, Dec 2018.

[15] Q. Li and D. Jiang, "Dc-link current analysis of three-phase 21-vsi considering ac current ripple," IET Power Electronics, vol. 11, no. 1, pp. 202-211, Nov 2017.

[16] L. Zhan, Y. Liu, and Y. Liu, "A clarke transformation-based dft phasor and frequency algorithm for wide frequency range," IEEE Trans. Smart Grid, vol. 9, no. 1, pp. 67-77, Jan 2018.

[17] T. Dragičević, "Model predictive control of power converters for robust and fast operation of ac microgrids," IEEE Trans. Power Electron., vol. 33, no. 7, pp. 6304-6317, Jul 2018.

[18] T. Dragicevic, C. Zheng, J. Rodriguez, and F. Blaabjerg, "Robust quasipredictive control of lcl-filtered grid converters," IEEE Trans. Power Electron., pp. 1-1, 2019.

[19] S. A. Richter and R. W. De Doncker, "Digital proportional-resonant (pr) control with anti-windup applied to a voltage-source inverter," in Proceedings of the 2011 14th European Conference on Power Electronics and Applications, Aug 2011, pp. 1-10. 